\title{
The psychological profile of female cancer patients undergoing radiotherapy: a preliminary study.
}

\author{
Mohammed M. J. Alqahtani ${ }^{1}$, Tamader Y. M. AL-Rammah ${ }^{2 *}$ \\ ${ }^{1}$ Department of Psychology, King Khalid University, Abha, Saudi Arabia \\ ${ }^{2}$ Radiologic Sciences, King Saud University, Saudi Arabia
}

\begin{abstract}
Although the prevalence and the risk factors of emotional distress among cancer patients are well documented, less is known in Saudi Arabia about such issue. The current study explores and describes that emotional distress symptom in diagnosed cancer patients during their radiation therapy. A total of 40 cancer patients, recruited from the largest oncology centre in Saudi Arabia, completed the study's questionnaire, which included the HADS and the fatigue symptom inventory.

Among cancer survivors, the prevalence of depression was $60 \%$, anxiety was $37.5 \%$, and fatigue was $77.5 \%$. Emotional distress, having any of the previous symptoms, was reported among $82 \%$ of the patients. Hierarchical regression analyses show having previous chemotherapy experiences and other predictors contributed modestly to variance in depression and fatigue models, compared to the predictor variable of having experiences with complementary intervention, which contributed significantly. Complementary intervention was the top predictor variable contributing to explaining emotional distress as a model.

This concurrent result may be helpful in developing clinical guideline strategies for psycho-oncology care to bridge the gap between the universal standard and the reality of health services.
\end{abstract}

Keywords: Emotional distress, Fatigue, Anxiety, Depression, Psycho-oncology, Radiotherapy, Cancer.

Accepted on January 18, 2018

\section{Introduction}

Radiation therapy is associated with physical and functional challenges that increase in severity during treatment and often persist long after treatment ends, leading to psychological complications that can affect the patient's quality of life [1]. Emotional distress is under recognised and under treated in cancer patients [2]. Cancer patients frequently report several psychological and treatment-related symptoms [3]. Depression, anxiety, and fatigue are viewed as a cluster of emotional distress symptoms commonly found in cancer patients $[4,5]$.

Depression is characterised by a loss of interest or pleasure in normal activities, with additional symptoms, including feelings of worthlessness and guilt, moodiness, concentration limitations, and changes in appetite, energy, and sleep. Anxiety could be characterised by autonomic arousal and fearfulness [6], which includes an emotional state comprising feelings of apprehension and tension [7]. Fatigue is one of the most common side effects of cancer and cancer-related treatments, characterizing by feelings of tiredness, weakness, and lacking energy [8].

\section{Radiation oncology In Saudi Arabia}

The total number of adult cancer incident cases reported to the Saudi Cancer Registry (SCR) was 14,796 (46.5\% male and $53.5 \%$ female) with a male to female ratio of $86: 100$. The median age of diagnosis was $58 \mathrm{y}$ for males and $51 \mathrm{y}$ for females [9]. Radiation therapy is usually delivered in outpatient clinics; patients attend five days a week for a period that can go up to eight weeks, depending on the cancer type and location. In Saudi Arabia, the Ministry of Health (MOH) has established comprehensive cancer centres. Most are within the existing modern and sophisticated health care service system and infrastructure. However, as in most developing countries, emotional distress among cancer patients is not the focus [10]. To some extent, psycho-oncology has been initially launched as a separate or even isolated service not well integrated with the practice of oncology across the country.

\section{Problems}

For cancer patients, health professionals, who do not routinely assess for emotional distress, might fail to detect that a patient needs psychosocial support [11]. Few studies in Saudi Arabia and other Arabic countries explore the psychological morbidity of cancer patients. Most studies were conducted in a palliative care clinic when cancer patients approached death [12-14]. 
Therefore, it is important to detect emotional distress and develop a supportive psycho-oncology program to help cancer patients during their treatment stages. Psycho-oncology recognises the importance of various psychological, social, and behavioural factors related to cancer prevention, cancer illness and interventions, and cancer survivorship that may influence cancer morbidity and mortality [15].

\section{Aim}

The current study attempts a preliminary scan of emotional distress in one of the most advanced tertiary hospitals, providing cancer care in Saudi Arabia, to note with overall observations on its status and devise future guidelines that emerge from such study. Identification of factors associated with poorer psychological outcomes in radiation therapy patients may aid the radiation oncology healthcare team in identifying patients in need of additional psychosocial support.

\section{Methodology}

Participants were approached in the radiation therapy waiting area of one of the largest oncology centres in Saudi Arabia. All participants were diagnosed with cancer and were undergoing radiation therapy. The aim and objective of the current study were explained to patients, who were then asked to complete the study's questionnaire. The questionnaire required 15-20 min to answer and included four parts: (1) patient demographics; (2) patient and family history; (3) psychological fatigue scale; and (4) hospital anxiety and depression scale (HADS). Approval of the research protocol was granted by the Medical Research Ethics Committee of King Saud University (KSU).

\section{HADS}

Depression and anxiety subscales of HADS were used as continuous variables to assess depression and anxiety. Patients with high scores, at or above the recommended threshold value of 11 on either subscale were categorised as cases [16]. HADS is frequently used in oncology studies and is found to report high sensitivity and specificity [17].
The fatigue symptom inventory was used to detect fatigue symptoms. Preliminary evidence of the reliability and validity of the fatigue symptom inventory has been reported for women with a variety of cancer diagnoses [18]. The fatigue symptom inventory was developed mainly with women undergoing or who had completed cancer treatment [19]. As it was used in previous studies [20], the current study categorised the results of the fatigue symptom as a moderate level of six and above.

\section{Data analysis}

The Statistical Package for the Social Sciences 22 (SPSS.22) program was used for analysis. For ease of analysis, most variables were grouped. To test the effect of the demographics of the sample, the study defined emotional distress as a high score on the depression, anxiety, and fatigue scales. Chi-square tests and Cross-tabulations were used to determine whether there was a significant difference between frequencies, and Cross-tabulations transferred the frequencies to categories. Only significant variables in the Chi-squared test from previous analyses were used in further analyses. Hierarchical regression was applied to detect to what extent significant variables in the Chi-squared test, time since diagnosis, previous chemotherapy, and complementary/alternative intervention contribute to the prediction of patients' depression, anxiety, and fatigue.

\section{Results}

Descriptive statistics among the continuous study variables are summarised in Table 1. The final sample included 40 outpatients, who received an external treatment type of radiotherapy. The period of radiation therapy treatment ranges from one to four weeks, whereas the mean time since diagnosis is about seven months. Based on the Chi-square test, no demographic differences between variables were seen, except for significant results in the variables of time since diagnosis $\left(\chi^{2}=5.19, \mathrm{p}<0.05\right)$, previous chemotherapy $\left(\chi^{2}=6.41, \mathrm{p}<0.001\right)$, and complementary/alternative intervention $\left(\chi^{2}=4.61, \mathrm{p}<0.05\right)$.

Table 1. Characteristics of cancer survivors at undergoing radiotherapy $(N=40)$.

\begin{tabular}{|c|c|c|c|c|c|}
\hline Characteristics & & $\mathbf{N}$ & $\%$ & $x^{2}$ & $\mathbf{P}$ \\
\hline \multirow[t]{4}{*}{ Mean age in years (SD) } & $18-25$ & 8 & 20 & 3.12 & \\
\hline & $26-45$ & 13 & 32.5 & & $\mathrm{n} / \mathrm{a}[3]$ \\
\hline & $46-65$ & 14 & 32.5 & & \\
\hline & $\geq 66$ & 5 & 12.5 & & \\
\hline \multirow[t]{3}{*}{ Education } & Illiteracy & 10 & 25.5 & 2.85 & $\mathrm{n} / \mathrm{a}$ \\
\hline & Less educated [1] & 14 & 32.5 & & \\
\hline & More educated [2] & 16 & 40 & & \\
\hline Marital status & Married & 32 & 80 & 1.33 & $\mathrm{n} / \mathrm{a}$ \\
\hline
\end{tabular}




\begin{tabular}{|c|c|c|c|c|c|}
\hline & \\
\hline & Unmarried & 7 & 17.5 & & \\
\hline & Widowed & 1 & 2.5 & & \\
\hline \multirow[t]{2}{*}{ Have children } & Yes & 29 & 72.5 & 1.1 & $\mathrm{n} / \mathrm{a}$ \\
\hline & No & 11 & 27.5 & & \\
\hline \multirow[t]{3}{*}{ Family history of cancer } & No & 24 & 60 & 2.03 & $\mathrm{n} / \mathrm{a}$ \\
\hline & Yes, first degree & 9 & 22.5 & & \\
\hline & Yes, second degree & 7 & 17.5 & & \\
\hline \multirow[t]{5}{*}{ Type of cancer } & Brain and head & 7 & 17.5 & 3.17 & $\mathrm{n} / \mathrm{a}$ \\
\hline & Breast & 19 & 47.5 & & \\
\hline & Uterus & 3 & 7.5 & & \\
\hline & Lymphoma & 9 & 22.5 & & \\
\hline & Other & 2 & 5 & & \\
\hline \multirow[t]{3}{*}{ Time since diagnosis } & $<6$ months & 13 & 32.5 & 5.19 & 0.04 \\
\hline & 7-12 months & 16 & 40 & & \\
\hline & $>13$ months & 11 & 27.5 & & \\
\hline \multirow[t]{7}{*}{ Complementary/alternative medicine } & Special diet & 5 & 12.5 & 6.41 & 0.01 \\
\hline & Herbal Arabic & 6 & 15 & & \\
\hline & Vitamins and supplements & 2 & 5 & & \\
\hline & Physical interventions & 3 & 7.5 & & \\
\hline & Spiritual practices & 21 & 52.5 & & \\
\hline & Folk remedies, healing & 4 & 10 & & \\
\hline & Chinese medication & 1 & 2.5 & & \\
\hline \multirow[t]{2}{*}{ Aware of cancer } & Yes & 37 & 92.5 & 0.8 & $\mathrm{n} / \mathrm{a}$ \\
\hline & No, patient-designated by proxy of his relative. & 3 & 7.5 & & \\
\hline \multirow[t]{3}{*}{ Previous treatment received } & Surgery only & 12 & 30 & 4.61 & 0.05 \\
\hline & Chemotherapy & 19 & 47.5 & & \\
\hline & Chemo+surgery & 9 & 22.5 & & \\
\hline
\end{tabular}

The results in Table 2 show the clusters of symptoms based on four categories: depression, anxiety, fatigue, and emotional distress. Cross-tabulations were used in Table 2 to cluster the prevalence of depression $(60 \%)$, anxiety $(37.5 \%)$, and fatigue
$(77.5 \%)$ and the combined type (depression+anxiety $=70 \%)$ and emotional distress (depressio+anxiety+fatigue $=82 \%$ ) among patients.

Table 2. Cross-tabulations and percentages comparing between depression, anxiety, fatigue, and psychological caseness (emotional distress).

\begin{tabular}{|c|c|c|c|c|c|c|c|c|c|}
\hline & & & \multicolumn{2}{|c|}{ Depression $(n=24) 60 \%$} & \multicolumn{2}{|c|}{ Anxiety $(n=15) 37.5 \%$} & $\begin{array}{l}\text { Combined type } \\
\text { +anxiety) }(n=28) 70 \%\end{array}$ & (Depression & \multirow{2}{*}{$\begin{array}{l}\begin{array}{l}\text { Any psychological } \\
\text { caseness }\end{array} \\
(+)(+)\end{array}$} \\
\hline & & & Negative (-) & Positive (+) & Negative (-) & Positive (+) & Negative (-) & Positive (+) & \\
\hline \multirow{2}{*}{$\begin{array}{l}\text { Fatigue } \\
77.5 \%\end{array}$} & $(n=31)$ & $(-)$ & 9 & 0 & 5 & 4 & 7 & 2 & $(n=33) 82.5 \%$ \\
\hline & & $(+)$ & 7 & 24 & 20 & 11 & 5 & 26 & \\
\hline
\end{tabular}

Hierarchical regression analyses are shown in Table 3, where four separate analyses were conducted. The predictor variable of time since diagnosis was entered at Step 1, followed by the predictor variables, previous chemotherapy and complementary intervention, in Steps 2 and 3, respectively. For the first analysis (depression model), changes in the time since 
diagnosis accounted for an average of $\mathrm{R}^{2}=0.15, \mathrm{p}<0.01$ and contributed to explaining depression among patients. Against our expectations, previous chemotherapy experiences contributed modestly to variance in depression, with about $\mathrm{R}^{2}=0.09$. Astonishingly, the predictor variable of experiences with complementary intervention contributed significantly $\left(\mathrm{R}^{2}=0.32, \mathrm{p}<0.001\right)$ to explaining depression. The results show less experience with complementary intervention led to an increase in the depression level. Overall, this model explained $56 \%$ of the variance of patients' depression.

Table 3. Hierarchical regression analysis: depression, anxiety, fatigue, and psychological caseness variables.

\begin{tabular}{|c|c|c|c|c|c|}
\hline Dependent variables & Predictor variables & Beta & $\mathbf{R}^{2}$ change & Total $\mathbf{R}^{2}$ & $F(d . f)$ \\
\hline & Analysis 1 & & & & \\
\hline \multirow[t]{4}{*}{ Depression } & Step 1: Time since diagnosis & $0.22^{*}$ & $0.15^{* *}$ & $0.56^{* * *}$ & 7.09 \\
\hline & Step 2: Previous chemotherapy & 0.16 & 0.09 & & $(6 ; 37)$ \\
\hline & Step 3: Complementary/alternative medicine & $-42^{* * *}$ & $0.32^{* * \star}$ & & \\
\hline & Analysis 2 & & & & \\
\hline \multirow[t]{4}{*}{ Anxiety } & Step 1: Time since diagnosis & 0.12 & 0.08 & $0.29^{*}$ & 6.22 \\
\hline & Step 2: Previous Chemotherapy & $0.31^{* * *}$ & $0.17^{* \star}$ & & $(6 ; 38)$ \\
\hline & Step 3: Complementary medicine & -0.08 & 0.04 & & \\
\hline & Analysis 3 & & & & \\
\hline \multirow[t]{4}{*}{ Fatigue } & Step 1: Time since diagnosis & $0.31^{* * *}$ & $0.16^{* *}$ & $0.52^{* * *}$ & 8.04 \\
\hline & Step 2: Previous Chemotherapy & $0.27^{\star \star \star *}$ & $0.17^{\star \star}$ & & $(5 ; 38)$ \\
\hline & Step 3: Complementary medicine & $-0.35^{* \star *}$ & $0.19^{* \star *}$ & & \\
\hline & Analysis 4 & & & & \\
\hline \multirow[t]{3}{*}{ Emotional distress } & Step 1: Time since diagnosis & $0.31^{*}$ & $0.14^{*}$ & $0.51^{* * *}$ & 11.27 \\
\hline & Step 2: Previous Chemotherapy & 0.13 & 0.09 & & $(5 ; 39)$ \\
\hline & Step 3: Complementary medicine & $-0.36^{\star \star}$ & $0.28^{\star \star}$ & & \\
\hline
\end{tabular}

In the anxiety model in the second analysis, the predictor variables of time since diagnosis and complementary intervention accounted for a few changes: $\mathrm{R}^{2}=0.08$ and $\mathrm{R}^{2}=0.04$, respectively. However, the predictor variable of previous chemotherapy contributed significantly $\left(\mathrm{R}^{2}=0.17\right.$, $\mathrm{p}<0.01)$ to explaining anxiety. Overall, this model explained $29 \%$ of the variance of patients' anxiety.

The third analysis was to explain the fatigue model. All of the three predictor variables from Step 1 to Step 3 contributed significantly $\left(\mathrm{R}^{2}=0.16, \mathrm{p}<0.01 ; \mathrm{R}^{2}=0.17, \mathrm{p}<0.01 ; \mathrm{R}^{2}=0.19\right.$, $\mathrm{p}<0.001)$ to explaining fatigue, respectively. Again, complementary intervention was the top predictor variable contributing to explain the fatigue model. Overall, this model explained $52 \%$ of the variance of patients' fatigue.

The final analysis (emotional distress) tries to explain depression, anxiety, and fatigue in one model. The model explains $51 \%$ of the variance. Once more, complementary intervention was the top predictor variable contributing to explaining the emotional distress model $\left(\mathrm{R}^{2}=0.28, \mathrm{p}<0.001\right)$.

\section{Discussion}

The present study provides an important sample of Saudi female cancer patients. It is the first investigation to explore representative data on depression, anxiety, and fatigue symptoms for all cancer types while receiving radiotherapy. It is also the first to examine psychological symptom clusters among cancer patients in Saudi Arabia, investigating complementary intervention influences.

\section{Depression only}

In our results, the prevalence of depression was higher than other Saudi studies [21]. The high rates of depression in the current study could be explained by including many cancers, whereas the previous Saudi study included only breast cancer patients. However, our high level of depression agrees with a more recent Saudi study [14], where depression was found in over $50 \%$ of cancer patients. Some studies reported more depressive symptoms were observed among women receiving chemotherapy [22], and through all cancer types, female patients showed higher levels of depression and anxiety than men [23]. The high levels of depression need to be seen in light of the descriptive data of our sample. The majority of our 
patients are in their first year after diagnosis, which could be linked to the high levels of depression [24].

\section{Anxiety only}

The level of anxiety in the present sample was lower than reported with depression and fatigue. However, patients could find it difficult to distinguish between anxiety and other psychological distress such as depression. Frequently, anxiety is accompanied by other emotional distresses, which could lead the cancer patient to report only one of them, resulting in more impact on their case. Another explanation is that cancer patients may understand anxiety as a normal symptom after a cancer diagnosis or even a constructive part of dealing with cancer [25]. Understanding the nature of the anxiety in cancer patients is crucial, because abnormal anxiety disrupts pharmacologic and psychological intervention [26].

\section{Fatigue}

In our results, the most common symptom was fatigue $(77.5 \%)$. Fatigue is one of the most common symptoms experienced by patients undergoing radiotherapy for cancer [20]. Several studies have noted patients define fatigue as the most distressing of the symptoms they experience during their cancer intervention [27]. Our findings on fatigue are also supported by previous Arabic studies, which highlighted that fatigue seems to be the most frequent symptom among Arabic cancer patients with a prevalence rate of about $80 \%[12,13]$.

\section{Clusters emotional distress}

The results of the current study display the clusters of emotional distress among cancer patients. These findings are supported by previous studies, showing strong links between depression and anxiety [28], and depression and fatigue, which may share the same pathophysiologic mechanisms [23,29], and symptom clusters of depression, anxiety, and fatigue in cancer patients [5]. Our results exhibit that depression and anxiety could be present or absent, but fatigue was usually present. Fatigue symptoms in our study appear as the dominant factor of emotional distress among cancer patients.

Frequently, fatigue is among the most distressing symptoms for most cancer patients and could continue for months or even years after completing cancer intervention [30]. Because of the naturalness of this symptom, fatigue could become the first cancer-related distress and remain as the last residual symptom.

Professionals increasingly identify that fatigue represents a significant consequence of cancer and its treatment that requires professional attention and intervention [8]. Unfortunately, most emotional distress, mainly fatigue, among Saudi cancer patients could be ignored, paying more efforts toward the biophysical side. The absence of psycho-oncology could play an essential role in the dual absence of psychometric assessments and psychological intervention. Even when emotional distress accrues, pharmacological intervention seems provided alone.
In a systematic and meta-analytic review [31], nonpharmacological interventions, mainly psychological intervention, are effective in reducing emotional distress through a wide spectrum of cancer populations. To follow the international recommendation of providing quality cancer care for the whole patient [32], health services for cancer patients should be delivered by a multidisciplinary team, including a psycho-oncologist, whom would be a key source of psychological support during and after patients receiving their cancer diagnosis and interventions [33].

Nowadays, cancer care has universally improved to a different standard of care and to a new model that provides cancer care as multidisciplinary for the whole patients. We need this integration in cancer care in Saudi Arabia.

\section{Complementary}

Interestingly, clear inverse relationships were seen between depression and fatigue with complementary interventions. Having more experience with complementary interventions was linked with lower levels of depression and fatigue and low emotional distress. Our findings show about $52 \%$ of the sample uses spiritual practices as a complementary intervention.

Spiritual practices were among the most common complementary interventions that patients held and practised, reflecting the importance of spirituality in Saudi society [34]. A Muslim frequently seeks spiritual healing, such as prayers and reading the Quran, for curing all illness [35]. A result of a recent meta-analysis study suggests greater spirituality is associated with better health improvement among cancer patients [36]. This result was highlighted to provide healthcare for cancer patients with all resources available, including spiritual resources, which encourage recovery, adjustment, and quality of life [37]. Future studies will aid our understanding of the relationships between spirituality and health outcomes in cancer patients in Saudi Arabia.

\section{Clinical implementation}

Our current findings highlight the importance of improving supportive care services for patients with cancer. Dynamic psychological services are needed for women with cancer, particularly in the first year after diagnosis and around the radiotherapy period.

The absence of problems from emotional distress after the diagnosis of cancer and during radiotherapy does not imply the absence of psychological difficulties. Rather, it could mean such hidden distress was not evaluated or managed. Improving psycho-oncology support might limit hidden distress in female cancer patients in Saudi Arabia.

\section{Conclusion}

Though a small sample, these result documents, for the first time, the profile of emotional distress among cancer patients undergoing radiotherapy in Saudi Arabia. The results show a need for routine psychological assessment and management of 
the hidden morbidity of psychological distress among cancer patients.

Additional effort is called for from healthcare professionals and research to improve psycho-oncology services for cancer patients and establish a clinical guideline for psycho-oncology care. Such a step is essential to nominate psychosocial oncology to find its place as a core service within the cancer care system nationally. Researchers in the field of cancer in Saudi Arabia could find the current data valuable in their efforts to improve methods for preventing or reducing psychological distress in patients undergoing radiotherapy.

\section{Limitations}

Acknowledged limitations of this descriptive study should be mentioned. Our study includes a relatively small number of participants, restricting the generalizability of our results. Data were also not collected regarding comorbid mental illnesses or receiving medications (e.g., antidepressants) that could contribute to feelings or absence of psychological distress.

In several studies, HADS was the main tool used to assess psychological distress among cancer patients; it appears to be a sensitive instrument. However, the choice of ideal threshold scores for cancer patients must be carefully considered [38]. The optimal threshold is one that minimises the variance between sensitivity and specificity. This optimal threshold for cancer patients in the Arabic population must be investigated in future studies.

\section{Disclosures}

The authors have no conflicts of interest to disclose. This study did not receive external funding.

\section{References}

1. Badr H, Gupta V, Sikora A, Posner M. Psychological distress in patients and caregivers over the course of radiotherapy for head and neck cancer. Oral Oncol 2014; 50: 1005-1011.

2. Bultz BD, Johansen C. Screening for distress, the 6th vital sign: where are we, and where are we going? Psycho Oncol 2011; 20: 569-571.

3. Lawrence DP, Kupelnick B, Miller K, Devine D, Lau J. Evidence report on the occurrence, assessment, and treatment of fatigue in cancer patients. Monographs Nat Cancer Inst 2004; 32: 40-50.

4. Dodd MJ, Miaskowski C, Lee KA. Occurrence of symptom clusters. Monographs Nat Cancer Inst 2004; 32: 76-78.

5. Brown LF, Kroenke K. Cancer-related fatigue and its associations with depression and anxiety: a systematic review. Psychosomatics 2009; 50: 440-447.

6. Goodenough B, Drew D, Higgins S, Trethewie S. Bereavement outcomes for parents who lose a child to cancer: are place of death and sex of parent associated with differences in psychological functioning? Psycho Oncol 2004; 13: 779-791.
7. Zhu L, Ranchor AV, Lee M, Garssen B, Almansa J, Sanderman R, Schroevers MJ. Comorbidity of depression, anxiety and fatigue in cancer patients receiving psychological care. Psycho Oncol 2016.

8. Hofman M, Ryan JL, Figueroa-Moseley CD, Jean-Pierre P, Morrow GR. Cancer-related fatigue: the scale of the problem. Oncologist 2007; 12: 4-10.

9. Cancer Incidence Report, Saudi Arabia 2013. Saudi Cancer Registry. June 2016, Available from http: // scr.org.sa/ reports.

10. Ezzat AA, Ibrahim EM, Raja MA, Al-Sobhi S, Rostom A, Stuart RK. Locally advanced breast cancer in Saudi Arabia: high frequency of stage III in a young population. Med Oncol 1999; 16: 95-103.

11. Jenkins V, Fallowfield L, Saul J. Information needs of patients with cancer: results from a large study in UK cancer centres. Br J Cancer 2001; 84: 48.

12. Alshemmari S, Ezzat H, Samir Z, Sajnani K, Alsirafy S. Symptom burden in hospitalized patients with cancer in Kuwait and the need for palliative care. Am J Hosp Palliat Med 2010

13. Ghosn M, Boutros C, Geara S, Kattan J, Nasr F, Chahine G. Experience with palliative care in patients with advanced cancer at a tertiary care hospital in a developing country. Support Care Cancer 2011; 19: 573-575.

14. Al-Shahri MZ, Eldali AM, Al-Zahrani O. Nonpain symptoms of new and follow-up cancer patients attending a palliative care outpatient clinic in Saudi Arabia. Ind $\mathrm{J}$ Palliat Care 2012; 18: 98.

15. Dunn J, Adams C, Holland J, Watson M. Reinforcing the role of psychosocial oncology in global cancer prevention: applying PsychoOncology research in programmes and practice. Psycho Oncol 2015; 24: 1217-1221.

16. Hopwood P, Howell A, Maguire P. Screening for psychiatric morbidity in patients with advanced breast cancer: validation of two self-report questionnaires. $\mathrm{Br} \mathrm{J}$ Cancer 1991; 64: 353.

17. Bjelland I, Dahl AA, Haug TT, Neckelmann D. The validity of the Hospital Anxiety and Depression Scale: an updated literature review. J Psychosom Res 2002; 52: 69-77.

18. Hann DM, Denniston MM, Baker F. Measurement of fatigue in cancer patients: further validation of the fatigue symptom inventory. Qual Life Res 2000; 9: 847-854.

19. Hann DM, Jacobsen PB, Azzarello LM, Martin SC, Curran SL, Fields KK, Greenberg H, Lyman G. Measurement of fatigue in cancer patients: development and validation of the Fatigue Symptom Inventory. Qual Life Res 1998; 7: 301-310.

20. Hickok JT, Roscoe JA, Morrow GR, Mustian K, Okunieff $\mathrm{P}$, Bole CW. Frequency, severity, clinical course, and correlates of fatigue in 372 patients during 5 weeks of radiotherapy for cancer. Cancer 2005; 104: 1772-1778.

21. Al-Zaben F, Sehlo MG, El-deek BS, Koenig HG. Depressive symptoms, correlates, and the marital 
relationship in women with breast cancer in Saudi Arabia. Women Health Open J 2015; 1: 53-62.

22. So WK, Marsh G, Ling WM, Leung FY, Lo JC, Yeung M, Li GK. Anxiety, depression and quality of life among Chinese breast cancer patients during adjuvant therapy. Eur J Oncol Nurs 2010; 14: 17-22.

23. Bower JE, Ganz PA, Aziz N, Fahey JL. Fatigue and proinflammatory cytokine activity in breast cancer survivors. Psychosomat Med 2002; 64: 604-611.

24. Henselmans I, Helgeson VS, Seltman H, de Vries J, Sanderman R, Ranchor AV. Identification and prediction of distress trajectories in the first year after a breast cancer diagnosis. Health Psychol 2010; 29: 160.

25. Andersen BL, Tewfik HH. Psychological reactions to radiation therapy: reconsideration of the adaptive aspects of anxiety. J Personal Soc Psychol 1985; 48: 1024.

26. Mystakidou K, Tsilika E, Parpa E, Sakkas P, Vlahos L. The psychometric properties of the Greek version of the statetrait anxiety inventory in cancer patients receiving palliative care. Psychol Health 2009; 24: 1215-1228.

27. Saligan LN, Lukkahatai N, Holder G, Walitt B, MachadoVieira R. Lower brain-derived neurotrophic factor levels associated with worsening fatigue in prostate cancer patients during repeated stress from radiation therapy. World J Biol Psych 2015; 1-7.

28. Chow E, Fan G, Hadi S, Wong J, Kirou-Mauro A, Filipczak L. Symptom clusters in cancer patients with brain metastases. Clin Oncol 2008; 20: 76-82.

29. Fox SW, Lyon D. Symptom clusters and quality of life in survivors of ovarian cancer. Cancer Nurs 2007; 30: 354-361.

30. Cella D, Davis K, Breitbart W, Curt G. Cancer-related fatigue: prevalence of proposed diagnostic criteria in a United States sample of cancer survivors. J Clin Oncol 2001; 19: 3385-3391.

31. Kangas M, Bovbjerg DH, Montgomery GH. Cancer-related fatigue: a systematic and meta-analytic review of nonpharmacological therapies for cancer patients. Psychol Bull 2008; 134: 700 .
32. Bultz BD, Travado L, Jacobsen PB, Turner J, Borras JM, Ullrich AW. 2014 Presidents plenary international PsychoOncology society: moving toward cancer care for the whole patient. Psycho Oncol 2015; 24: 1587-1593.

33. Kubota Y, Okuyama T, Uchida M, Umezawa S, Nakaguchi T, Sugano K, Ito Y, Katsuki F, Nakano Y, Nishiyama T, Katayama Y. Effectiveness of a psycho-oncology training program for oncology nurses: a randomized controlled trial. Psycho Oncol 2016; 25: 712-718.

34. Alqahtani MM, Salmon P. Cultural influences in the aetiological beliefs of Saudi Arabian primary care patients about their symptoms: the association of religious and psychological beliefs. J Religion Health 2008; 47: 302-313.

35. Alqahtani MM. Understanding the sociocultural health belief model influencing health behaviors among Saudi stroke survivors. Neurosci Med 2015; 6: 149.

36. Salsman JM, Fitchett G, Merluzzi TV, Sherman AC, Park CL. Religion, spirituality, and health outcomes in cancer: A case for a metaanalytic investigation. Cancer 2015; 121: 3754-3759.

37. Cotton SP, Levine EG, Fitzpatrick CM, Dold KH, Targ E. Exploring the relationships among spiritual well-being, quality of life, and psychological adjustment in women with breast cancer. Psycho Oncol 1999; 8: 429-438.

38. Carey M, Noble N, Sanson-Fisher R, Mackenzie L. Identifying psychological morbidity among people with cancer using the hospital anxiety and depression scale: time to revisit first principles? Psycho Oncol 2012; 21: 229-238.

\section{*Correspondence to}

Tamader Y. M. AL-Rammah

Radiologic Sciences

King Saud University

Saudi Arabia 